\title{
Avaliação de procedimentos rotineiros na suinocultura como métodos estimativos de estresse mediante a aplicação da vocalização e velocidade de manejo
}

\author{
Evaluation of routine procedures in swine production as estimative \\ methods of stress through the application of vocalization and speed \\ of handling
}

\begin{abstract}
Gisele Dela Ricci ${ }^{1,2 *}$, Cristiane Gonçalves Titto ${ }^{2}$, Késia Oliveira da Silva Miranda ${ }^{3}$, Patricia Nardin Berto ${ }^{4}$, Evaldo Lencioni Titto $^{2}$ e Vera Letticie de Azevedo Ruiz ${ }^{5}$
\end{abstract}

\footnotetext{
${ }^{1}$ Universidade de São Paulo, Faculdade de Zootecnia e Engenharia de Alimentos, Programa de Pós-Graduação em Zootecnia. Pirassununga, SP, Brasil.

2 Universidade de São Paulo, Faculdade de Zootecnia e Engenharia de Alimentos, Laboratório de Biometeorologia e Etologia. Pirassununga, SP, Brasil.

3 Universidade de São Paulo, Escola Superior de Agricultura "Luiz de Queiroz", Departamento de Engenharia de Biossistemas. Piracicaba, SP, Brasil.

${ }^{4}$ Universidade de São Paulo, Faculdade de Medicina Veterinária e Zootecnia, Programa de Pós Graduação em Nutrição e Produção Animal. Pirassununga, SP, Brasil.

${ }^{5}$ Universidade de São Paulo, Faculdade de Zootecnia e Engenharia de Alimentos, Departamento de Medicina Veterinária. Pirassununga, SP, Brasil.
}

*Autor para correspondência:
giseledelaricci@usp.br
Conflitos de Interesse:
Os autores declaram não ter
conflito de interesse
Licença:
Artigo publicado em acceso aberto
sob uma licença Creative
Commons CC-BY
Histórico:
Recebido: $02 / 03 / 18$;
Aceito: $01 / 07 / 19$
Período de publicaçao:
Julho-Dezembro de 2019

Julho-Dezembro de 2019

\section{RESUMO}

Vocalização e comportamento são métodos não invasivos de análise de bem-estar animal. O objetivo deste estudo foi avaliar o bem-estar de leitões durante as práticas de mossa australiana, desgaste de dentes e castração, a partir de vocalizações e aspectos comportamentais dos suínos. Para tal, foram utilizados 281 leitões, com aferições da intensidade máxima e mínima do ruído emitido pelos leitões e o tempo de realização. 0 comportamento dos leitões foi obtido de acordo com a atividade desempenhada durante o manejo e contenção. Para análise comportamental um etograma específico foi utilizado. A comparação de médias foi realizada pelo teste-T e PDIFF. A castração foi a prática com maior média de tempo de realização, seguida pelo desgaste de dentes e mossa. Os maiores valores de média de intensidade mínima e máxima foram encontrados para a castração de machos, no processo lento. Observou-se que o sexo não influenciou no tempo em que a mossa e o desgaste de dentes foram realizados, mas durante os mesmos o comportamento de esquiva foi o mais observado $(P<0,05)$, com baixa frequência na castração. Portanto, pode-se concluir que os procedimentos ocasionaram dor, caracterizada por demonstrações vocais e de comportamento negativo.

Palavras-chave: bem-estar, castração, desgaste dos dentes, mossa australiana, suínos.

\begin{abstract}
Vocalization and behavior are non-invasive methods of animal welfare analysis. The objective of this study was to evaluate the welfare of piglets during Australian ear notching, tooth wear and castration, from vocalizations and behavioral aspects of pigs. For this, 281 piglets were used, with measurements of the maximum and minimum intensity of the noise emitted by the piglets and the time of accomplishment. The behavior of the piglets was obtained according to the activity performed during handling and containment. For behavioral analysis a specific etogram was used. The comparison of means was performed by T-test and PDIFF. The castration was the practice with the highest average of time of accomplishment, followed by the wear of teeth and ear notching. The highest mean values of minimum and maximum intensity were found for male castration in the slow process. It was observed that sex did not influence the time when the ear notching and tooth wear were performed, but at the same time the avoidance behavior was the most observed ( $P$ $<0.05)$, with low frequency in castration. Therefore, it can be concluded that the procedures caused pain, characterized by vocal demonstrations and negative behavior. Key words: welfare, castration, teeth wear, Australian ear notching, swine.
\end{abstract}




\section{INTRODUÇÃO}

O mercado consumidor de produtos de origem animal tem direcionado maior atenção à ciência do bem-estar, buscando maior entendimento e transparência nas formas de produção, rejeitando práticas de manejo e alojamento que agridam a fisiologia e etologia dos animais (Poletto, 2009), como mossagem australiana, caudectomia, corte ou desgaste de dentes e castração sem anestesia.

A análise do bem-estar animal é obtida a partir de critérios comportamentais, com apresentação de estereotipias; fisiológicos com aumento das concentrações de cortisol, da frequência cardíaca e respiratória, respostas do sistema imunológico e a sanidade; e ambientais, com aumento dos níveis de ruídos (pressão sonora), da temperatura e umidade do ar (Baptista, Bertani é Barbosa, 2011; Broom e Molento, 2004).

A reavaliação das práticas dentro da produção animal, por meio de análises das necessidades e estados físicos e emocionais, identificação de problemas técnicos que causem dor e sofrimento e a recomendação de alterações de modelos e procedimentos que almejem respeito e ética com os animais é a principal base para o surgimento e comprometimento da ciência do bem-estar animal (Ramos, 2006).

Entre os métodos de mensurar o bem-estar animal, a vocalização tem-se mostrado eficiente (Weary \& Fraser,1997; Marx, Horn, Thielebein, Knubel \& Borell, 2003; Manteuffel, Puppe \& Schön, 2004), por ser um método não invasivo, uma vez que não interfere na expressão natural do comportamento, auxiliando na interpretação de reações comportamentais (Dunkan, 2005).

A vocalização é utilizada como método para se expressar sendo notada e utilizada como critério desde as primeiras semanas de nascido (Hessing, Hagelso, Van Beek, Wiepkema, Schouten, \& Krukow, 1993). Essa possui características individuais, sendo um importante método de comunicação entre indivíduos da mesma espécie (Grandin, 1998). Quando emitidas, em situações de estresse, as vocalizações atuam como indicador de qualidade de vida e como uma estimativa instantânea do estado emocional dos animais (Düpjan, Schön, Puppe, Tuchscherer \& Manteuffel, 2008).
Suínos apresentam vasto repertório de vocalizações que permitem a atração de parceiros, advertir a presença ou intimidar agressores. O estudo dos sons emitidos por suínos tem sido avaliado em diferentes idades, com identificação de situações de comunicação e estresse (Kasanen \& Algers, 2002), de conflitos (Weary \& Fraser, 1995; Talling, Lines, Wathes \& Waran, 1998; Marchant, Whittakerb \& Broom, 2001), relacionando tempo de expressão e frequência de realização. Vocalizações de baixa tonalidade, como grunhidos, são utilizadas para contatos sociais, com membros do mesmo grupo, à medida que, altas tonalidades, com semelhanças a gritos, são mais utilizadas em estados de excitação (Schrader \& Todt, 1998).

De acordo com a importância de se reavaliar os procedimentos realizados com suínos em seus primeiros dias de nascido, este estudo objetivou analisar o bem-estar de leitões durante as práticas de mossa australiana, desgaste de dentes e castração, sem anestesia, a partir de estimativas de vocalizações com análises comportamentais dos suínos e dos funcionários.

\section{MATERIAL E MÉTODOS}

O estudo foi conduzido nas instalações de maternidade do Setor de Suinocultura da Prefeitura, na Universidade de São Paulo, Campus Fernando Costa, em Pirassununga, estado de São Paulo. O local encontra-se em altitude de 340 metros, latitude sul de $21^{\circ} 80^{\prime} 00^{\prime \prime}$ e longitude oeste de 4725'42", clima Cwa (clima temperado úmido com Inverno seco e Verão quente) com temperaturas anuais mínimas médias de $13^{\circ} \mathrm{C}$ e máximas de $31^{\circ} \mathrm{C}$, segundo Köppen (Köppen, 2011).

Foram utilizadas 26 fêmeas, F1, Landrace x Large White e 281 leitões, sendo 154 fêmeas e 127 machos, em sistema de confinamento com celas parideiras para leitões até o quarto dia após o nascimento, com transferência para o sistema de semiconfinamento, em baias individuais nas quais não há gaiolas para as fêmeas e os leitões possuem acesso exclusivo do escamoteador. Os procedimentos avaliados foram mossa australiana (1), desgaste dos dentes (2) e castração (3).

Para isso, os leitões foram retirados em caixas plásticas das baias com gaiolas, local onde se somam cinco áreas, das quais há uma distância da sala de manejo de 1 metro da primeira até 25 
metros da última, onde foram realizados os procedimentos.

A mossa australiana (1) foi realizada nas extremidades das orelhas, com a numeração de acordo com a quantidade de animais nascidos, utilizando-se um alicate marcador de orelha de 30 $\mathrm{cm}$ em forma de $U$, mantendo-se livres as duas orelhas, fazendo com que o animal permaneça imobilizado. Para o desgaste dos dentes (2), o leitão foi contido pela cabeça, onde o dedo indicador foi inserido na cavidade bucal, junto a gengiva, afastando os lábios dos dentes que serão raspados. Foi utilizado um desbastador de dentes da marca Dremel, em nível máximo de rotação. Para o manejo da castração (3), o funcionário delimitou o animal em um pequeno espaço, com os membros posteriores voltados para cima, fazendo com que a bolsa escrotal fique livre para o manejo.

A técnica de castração utilizada pela granja foi via escrotal, sem aplicação de anestesia ou analgesia, na qual uma incisão de aproximadamente $2 \mathrm{~cm}$ expõe os testículos, para remoção por corte ou tração. O corte foi realizado por bisturi com aplicação de antissépticos locais para cicatrização (Sobestiansky, Wents, Silveira, é Sesti, 2003; Prunier et al., 2006).

As práticas com os leitões ocorreram no segundo dia após o nascimento, sendo realizadas diariamente por técnicos da granja seguindo o protocolo descrito. Nesse estudo, apenas animais sadios participaram dos procedimentos. Aqueles que se apresentavam enfermos ou muito pequenos em relação aos demais foram retirados do estudo e permaneceram em quarentena até a melhora do quadro.

Durante a realização dos procedimentos foram aferidas a intensidade máxima e mínima em decibéis ( $\mathrm{dB}$ ) do ruído emitido pelos leitões, por decibelímetros dataloggers (MINIPA, MSL1352C) posicionado a uma distância de, no máximo, $20 \mathrm{~cm}$ da cavidade bucal dos leitões (Figura 1).

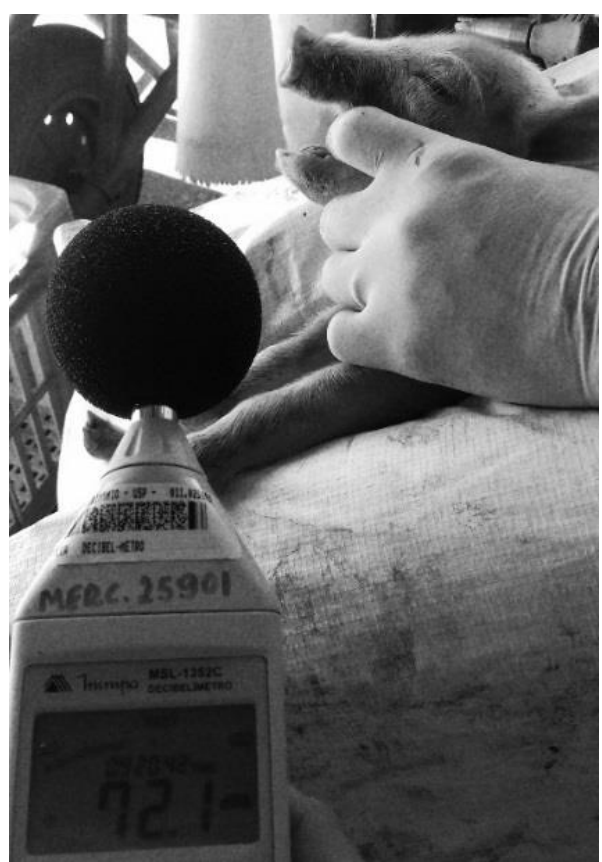

Figura 1. Contenção de leitão para a prática de desgaste de dentes com obtenção de intensidade mínima e máxima de ruído emitido com auxílio do decibelímetro. Fonte: Elaboração própria

O tempo de realização de cada prática foi mensurado individualmente a partir de um cronômetro digital, utilizado do momento em que o procedimento tinha início até a finalização deste. A velocidade de realização foi classificada como rápida, normal e lenta de acordo com os dados obtidos durante as práticas (Tabela 1 ).

Tabela 1. Classificação dos intervalos de tempos utilizados para mensurar a velocidade do manejo durante as práticas no segundo dia após o nascimento dos leitões.

\begin{tabular}{|c|c|c|c|}
\hline \multirow{2}{*}{ Procedimento } & \multicolumn{3}{|c|}{ Velocidade (s) } \\
\hline & Rápido & Normal & Lento \\
\hline Mossa Australiana & $<10,1$ & 10,2 a 18,7 & $>18,8$ \\
\hline Desgaste de dentes & $<10,3$ & 10,4 a 20,8 & $>20,9$ \\
\hline Castração & $<13,2$ & 13,3 a 30,9 & $>31$ \\
\hline
\end{tabular}

Fonte: Elaboração própria 
O comportamento dos leitões frente às três práticas foi realizado de acordo com o desenvolvimento dos leitões durante o manejo da contenção dentro do tempo obtido.

Confeccionou-se um etograma específico, com data e horário da avaliação, prática executada, manejador responsável e comportamentos agressivos, de esquiva, positivo, nenhum e outros dos leitões (Tabela 2).

Foi realizada correlação do sexo, peso e a velocidade de realização das práticas e o efeito entre tempo de realização dos leitões e intensidade do grito emitido pelos lactentes pelo SAS (2017). A comparação de médias foi realizada pelo teste-T e PDIFF.

Tabela 2. Etograma utilizado para avaliação do comportamento e intensidade da vocalização dos leitões durante a realização das práticas de manejo.

\begin{tabular}{cr}
\hline Comportamento & Descrição \\
\hline Agressivo & Tentativa de morder ou avançar no manejador \\
Esquivar & Tentativa de fuga da forma de contenção durante o manejo \\
Positivo & Tentativa de afago com o manejador \\
Nenhum & Nenhuma atividade, permanecer imóvel e sem vocalizar \\
Outro & Qualquer outra atividade que não esteja descrita nos comportamentos anteriores \\
\hline
\end{tabular}

Fonte: Elaboração própria

\section{RESULTADOS E DISCUSSÃO}

Nesse estudo, os leitões foram retirados das celas parideiras e encaminhados à sala de manejo. A localização da sala de manejo estava próxima às baias onde foram alojadas as matrizes e de acordo com a literatura, pela proximidade, os leitões foram capazes de ouvir aos chamados da mãe, devido à separação (Weary, Appleby \& Fraser, 1999) e por isso puderam reagir executando um tipo de chamado especifico, devido à frustração (Lewis, 1999). Suínos podem detectar sons na faixa de 20 a $20.000 \mathrm{~Hz}$, com deslocamento de ultrassom, e uma faixa de detecção de frequência razoável de 42 Hertz a 40,5 quilohertz com maior sensibilidade de $250 \mathrm{~Hz}$ a 16 kHz (Heffner \& Heffner, 1990). Puppe, Schön, Tuchscherer \& Manteuffel (2003), indicaram que leitões de diferentes leitegadas, ao ouvirem a gravação da vocalização da sua mãe, escolhiam permanecer mais próximos à fonte de vocalização da própria mãe em relação a de outras porcas.

A média de tempo para realização das práticas foi maior para a castração (3), seguida pelo desgaste dos dentes (2) e pela mossa australiana (1), com médias de 24,21, 16,03 e 15,05 segundos, respectivamente $(P<0,001)$. A prática de castração apresentou maiores valores de tempo para a realização nas três classificações de velocidade (lenta, normal e rápida), com 56, 30 e 13 segundos, apresentando também as maiores médias mínimas, nas três velocidades sendo 31 , 13,8 e 8 segundos.

Leidig, Hertrampf, Failing, Schumann, \& Reiner (2009) indicou que procedimentos cirúrgicos como corte de dentes e cauda, podem perdurar de 20 a 70 segundos, e que em um tempo médio de 45 segundos, o investimento em energia, devido à dor ou a fome por exemplo, pode ser de até 342,09 kcal. Grunhidos individuais em suínos podem durar, em média, de 0,13 a 2 segundos, considerando a média de 1 segundo por expressão vocal, como medo, com um gasto energético especifico para diferentes expressões, variando de 0,056 a 0,463 kcal, o que acarreta perdas produtivas importantes ao longo do sistema produtivo (Marchant et al., 2001). A dor ou muita dor pode gerar expressões de 3,6 e 3,7 vezes mais tempo de duração do que as relacionadas as condições normais, como grunhidos isolados e simples que estão associados a comportamentos exploratórios dos suínos (Kasanen \& Algers, 2002; Cordeiro, Nääs, Da Silva é De Moura, 2008).

A intensidade mínima do grito durante as práticas com menor e maior frequência foram obtidas na classificação lenta e rápida da castração com 58,8 e 103,0 dB (Tabela 3). Cordeiro, Nääs, Oliveira, Violaro, \& Almeida (2012), buscando estimar o nível de dor pela expressão vocal de suínos, avaliaram procedimentos de marcação, caudectomia e 
castração, em leitões na fase de maternidade, encontrando que a intensidade do som aumenta continuamente de normal $(70,41 \mathrm{~dB})$ para marcação $(77,64 \mathrm{~dB})$, depois para caudectomia $(88,31 \mathrm{~dB})$ e castração $(87,39 \mathrm{~dB})$, indicando que o aumento da vocalização do suíno segue o aumento do nível de dor. Suínos submetidos a estresse diversos, mesmo na ausência de dor e dependendo do estímulo, duração e grau do estresse, podem mudar de maneira diferente, intensidade do sinal das suas expressões vocais (Moi et al., 2015).

A velocidade classificada como rápida, apresentou, para todas as práticas, valores acima dos estabelecidos como normais $(70,41 \mathrm{~dB})$ Cordeiro et al. (2012) (Tabela 3). Foi descrito que a velocidade com que os procedimentos foram realizados pode causar lesões e dor nos leitões. Dela Ricci et al. (2017) avaliando a velocidade de realização do desgaste de dentes e a experiência dos manejadores ao realizar o manejo encontrou que o manejador com maior experiência causa menos lesões, mas que em maior velocidade, o manejador causou maior número de lesões de cavidade bucal dos animais.

No entanto, a velocidade lenta, pode apresentar problemas relacionados a redução do conforto dos animais e consecutivo aumento da vocalização mínima e máxima, explicado, principalmente, pelo método de contenção empregado para a realização dos três procedimentos avaliados. A prática classificada normal apresentou valores de intensidade mínima abaixo dos definidos como normais, no entanto apresentou níveis elevados para a intensidade máxima, indicando que todas as velocidades classificadas apresentaram alterações e possível dor e desconforto nos leitões (Tabela 3).

Tabela 3. Níveis de intensidade mínima e máxima de acordo com a classificação de velocidade para procedimentos de mossa australiana, desgaste de dentes e castração de leitões.

\section{Procedimento Velocidade Intensidade mínima Intensidade máxima}

\begin{tabular}{cccc}
\hline & Lenta & $62,09 \pm 3.064$ & $93,68 \pm 5.172$ \\
Mossa Australiana & Normal & $66,94 \pm 1.479$ & $99,73 \pm 2.497$ \\
& Rápida & $69,50 \pm 3.026$ & $101,79 \pm 5.108$ \\
\hline \multirow{2}{*}{ Desgaste de dentes } & Lenta & $59,69 \pm 3.234$ & $100,84 \pm 5.460$ \\
& Normal & $60,65 \pm 1.454$ & $100,15 \pm 2.460$ \\
& Rápida & $71,51 \pm 3.160$ & $90,02 \pm 5.334$ \\
\hline Castração & Lenta & $58,84 \pm 5.157$ & $103,06 \pm 8.706$ \\
& Normal & $62,12 \pm 2.170$ & $101,31 \pm 3.663$ \\
& Rápida & $65,68 \pm 5.157$ & $96,89 \pm 8.709$ \\
\hline
\end{tabular}

Fonte: Elaboração própria

Apesar das fêmeas terem apresentado peso médio inferior ao peso dos machos, com 1,674 kg e 1,791 kg, respectivamente, o peso dos leitões não influenciou na intensidade máxima e mínima do grito emitido durante a realização das três práticas.

O sexo não influenciou no tempo em que os procedimentos foram realizados. A média de tempo de realização total das duas práticas para as fêmeas foi de 18,33 minutos e para os machos de 18,54 minutos $(P>0,001)$. As médias de intensidades mínimas encontradas para as fêmeas, nas duas práticas, foi de $63,75 \mathrm{~dB}$ e para os machos de 64,47 $d B \quad(P>0,001)$. Em relação às intensidades máximas, para as fêmeas foram encontrados valores de 97,72 dB e de 99,50 dB para machos $(P>0,001)$.

O sexo dos animais e prática realizada com leitões não influiu na apresentação de comportamentos agressivos no momento do manejo ( $P>0,001)$. Ao sentir dor, o animal buscou eliminá-la a partir de posturas ou comportamentos, como por exemplo agressivos e estereotipados. Entretanto, caso não consiga, inicia-se a apresentação de respostas de 
má adaptação, permanecendo em estresse (Andrade, Correia, Pinto e Oliveira, 2002).

Durante o manejo de mossa, desgaste de dentes e castração, comprovadamente os leitões tentam esquivar-se dos objetos e manejadores, sendo o comportamento mais expresso pelos lactentes $(P<0,001)$. Notou-se que ou os animais estavam tentando a fuga ou permaneciam parados, sem esboçar nenhuma atividade corporal e vocal $(P<0,001)$. O comportamento agressivo realizado em baixa frequência $(0,3 \%)$ nas práticas 1 e 2 , não foi apresentado por leitões na prática de castração, explicado pela forma com que os leitões são contidos pelos manejadores, gerando imobilização. O comportamento positivo e outros não foram observados nas três práticas de manejo. Segundo Boissy et al. (2007) a manifestação de comportamentos específicos se estabelece como indicativo de desconforto ou frustração dos animais, geralmente pela privação de estímulos ambientais ou por circunstâncias diferentes dos habituais, como o manejo de contenção.

\section{CONCLUSÕES}

Diante dos resultados obtidos pôde-se concluir que procedimentos como mossa australiana, desgaste de dentes e castração são métodos que ocasionam dor, caracterizada por demonstrações vocais e de comportamento, além do prejuízo ao bem-estar integral dos suínos na maternidade.

Extinguir tais práticas requer treinamento de pessoal e conhecimento técnico sobre novos métodos de marcação, de eliminação de problemas com a mamada e sabor na carne. Priorizar o bemestar animal, diante às necessidades econômicas, tornar-se-á, em breve, pré-requisito para consumo de carne, levando os meios produtivos a se adaptarem às novas vertentes estipuladas.

\section{AGRADECIMENTOS}

A CAPES pelo consentimento do auxílio financeiro e desenvolvimento e a Universidade de São Paulo, pelo apoio ao desenvolvimento da pesquisa.

\section{REFERÊNCIAS BIBLIOGRÁFICAS}

Andrade, A., Correia, Pinto, S.C. é Oliveira, R.S. de (2002). Animais de Laboratório: criação e experimentação. Rio de Janeiro, Brazil: Editora FIOCRUZ, 388 p.
Baptista, R.I.A.A., Bertani, G. R. é Barbosa, C. N. (2011). Indicadores do bem-estar em 373 suínos. Ciência Rural, 41(10), pp.1823-1830.

Boissy A., Manteuffel, G., Jensen, M.B., Moe, R.O., Spruijt, B., Keeling, L.J., ... Aubert, A. (2007). Assessment of positive emotions in animals to improve their welfare. Physiology \& Behavior, Haren ,92(3), 375-397.

Broom, D.M. é Molento, C.F.M. (2004). Bem-estar animal: conceitos e questões relacionadas Revisão. Archives of Veterinary Science, 9(2), 1-11.

Cordeiro, A.F.D.S., Nääs, I.D.A., Da Silva, W.T., é De Moura, D.J. (2008). Medida de vocalização de suínos (Sus scrofa) como um indicador de gasto energético. Revista Brasileira de Engenharia de Biossistemas, 2(2), 143-152.

Cordeiro, A.F.S., Nääs, I. A., Oliveira, S. R. de M., Violaro, F. \& Almeida, A. C. M. (2012). Efficiency of distinct data mining algorithms for classifying stress level in piglets from their vocalization. Engenharia Agrícola, Jaboticabal, 32(2), 208-216.

Dela Ricci, G., Titto, C.G., Berto, P.N., Tonon, E., Titto, E.L. é Santos, J.V. (2017). Influência do treinamento de manejadores no aparecimento de lesões na cavidade bucal de leitões após pratica de desgaste de dentes. VII Brazilian Congress of Biometeorology, Ambience, Behaviour and Animal Welfare. Anais SBBiomet, Sociedade Brasilera de Biometeorología, 67-98p.

Dunkan, I.J.H. (2005). Science-based assessment of animal welfare: farm animals. Revue Scientifique et Technique-Office International des Épizooties, 24(2), 483-492.

Düpjan, S., Schön, P., Puppe, B., Tuchscherer, A. \& Manteuffel, G. (2008). Differential vocal responses to physical and mental stressors in domestic pigs (Sus scrofa). Applied Animal Behaviour Science, $114,105-115$.

Grandin, T. (1998). The feasibility of using vocalization scoring as an indicator of poor welfare during slaughter. Applied Animal Behaviour Science, 56(2), 121-8.

Heffner, R. S. \& H. E. Heffner. (1990). Vestigial hearing in a fossorial mammal, the pocket gopher 
(Geomys bursarius). Hearing Research, 46(1), 239252.

Hessing, M.J.C., Hagelso, A.M., Van Beek, J.A.M, Wiepkema, R. P., Schouten, W. G. P., \& Krukow, R. (1993). Individual behavioural characteristics in pigs. Applied Animal Behaviour Science., 37(4), 285-295.

Kasanen, S. \& Algers B. (2002). A note on the effects of additional sow gruntings on suckling behaviour in piglets. Applied Animal Behaviour Science, 72(2), 93-101.

Köppen: Clima dos Municipios Paulistas. (2011). Consultado 03 jan. 2018. Disponible: http://www.cpa.unicamp.br/outras-

informacoes/clima-dos-municipios-paulistas.html.

Leidig, M.S., Hertrampf, B., Failing, K., Schumann, A. \& Reiner, G. (2009). Pain and discomfort in male piglets during surgical castration with and without local anesthesia as determined by vocalization and defence behaviour. Applied Animal Behaviour Science, 116(2), 174-178.

Lewis, N. J. (1999). Frustration of goal-directed behaviour in swine. Applied Animal Behaviour Science, 64, 19-29.

Manteuffel, G., Puppe, B. \& Schön, P.C. (2004). Vocalization of animals as a measure of 429 welfare. Applied Animal Behaviour Science, 88, 163-182.

Marchant, J. N., Whittakerb, X. \& Broom, D.M. (2001). Vocalisations of the adult female domestic pig during a standard human approach test and their relationships with behavioural and heart rate measures. Applied Animal Behaviour Science, $72(2), 23-39$.

Marx, G., Horn, T., Thielebein, J., Knubel, B. \& Borell, E. (2003). Analysis of pain related vocalization in young pigs. Journal of Sound and Vibration, 266, 687-698.

Moi, M. Nääs, I. A., Caldara, F. R., Paz, I. C. L. A., Garcia, R. G., Cordeiro, A. F. S., \& Seno, L. O. (2015). Vocalização como indicativo do bem-estar de suínos submetidos a situações de estresse.
Arquivo Brasileiro de Medicina Veterinária e Zootecnia, 67(3), 837-845.

Poletto, R. (2009). Bem-Estar Animal. (Série Especial). Consultado 03 jan. 2018. Disponible http://www.suino.com.br/SanidadeNoticia.aspx?co digoNot $=z$ SoHh $5 f 8$ w90 $=$ \&title $=$ SE

Prunier, A., Bonneau M., Von Borell, E.H., Cinotti, S., Gunn, M., Fredriksen, B., \& Velarde, A. (2006). $A$ review of the welfare consequences of surgical castration in piglets and evaluation of non-surgical methods. Animal Welfare, 15(3), 277-289.

Puppe, B., Schön, P.C., Tuchscherer, A. \& Manteuffel, G. (2003). The influence of domestic piglets (Sus scrofa) age and test experience on the preference for the replayed maternal nursing vocalization in a modified open- field test. Acta Ethologica, 5(2), 123-129.

Ramos, J.B. (2006). Bem estar animal: A ciência de respeito aos animais. Informativo do instituto Ecológico Aqualung, 68: 4-6.

Schrader, L., Todt, D. (1998). Vocal quality is correlated with levels of stress hormones in domestic pigs. Ethology, Berlim, 104(10), 859-876.

Sobestiansky, J., Wents, I., Silveira, P.R.S é Sesti, L.A.C. (2003). Suinocultura Intensiva. 1. Brasília, Brazil: Editora Embrapa, 36p.

Talling, J. C., Lines, J. A., Wathes, C. M. \& Waran, N. K. (1998). The acoustic environment of the domestic pig. Journal of Agricultural Engineering Research, Silsoe, 71, 1-12.

Weary, D. M., Appleby, M. C. \& Fraser, D. (1999). Responses of piglets to early separation from the sow. Applied Animal Behaviour Science, Londres, 63(4), 289-300.

Weary, D. M. \& Fraser, D. (1995). Calling by domestic piglets: Reliable signals of need. Animal Behavior, 50, 1047-1055.

Weary, D.M. \& Fraser, D. (1997). Vocal response of piglet to weaning: effect of piglet age. Applied Animal Behaviour Science, 54, 153-160. 